\title{
A NOTE ON THE INTERSECTION OF FREE MAXIMAL IDEALS
}

\author{
STEWART M. ROBINSON
}

(Received 3 December 1967, revised 21 July 1968)

In [2] we proved that if $X$ admits a complete uniform structure, the intersection of the free maximal ideals in $C(X)$ is precisely $C_{K}(X)$, the ring of functions with compact support. In the present paper we are able to sharpen this result somewhat and give necessary and sufficient conditions on a space $X$ so that this conclusion holds. Both our previous result and that of Kohls for $p$-spaces follow as special cases of our theorem.

We employ the same terminology and conventions as in [1] and [2]. In particular, each space $X$ is assumed to be completely regular, and to each point $p \in \beta X-X$, we associate the free maximal ideal

$$
M^{p}=\left\{f \in C(X): p \in c l_{\beta X} Z_{\rho}\right\} .
$$

We recall the facts that if $M^{p}$ is real, the associated $Z$-ultrafilter $Z\left[M^{p}\right]=\left\{Z_{f}: f \in M^{p}\right\}$ is closed under countable intersections, and that if $M^{p}$ is hyper-real, there is some $h \in C(X)$ such that $c l_{\beta X}\{x: h(x) \geqq n\}$ is a neighborhood of $p$ for each $n \in N$.

Finally, we shall denote by $S_{f}$ the support $\left(=c l_{X}\left(X-Z_{f}\right)\right)$ of $f \in C(X)$.

The following lemma sharpens that of [2].

Lemma. If $f \in C(X)$ and if $X-Z_{f}$ contains a subset $A$ which is a closed, non-compact, $C$-embedded subset of $S_{f}$, then $f$ fails to belong to some free maximal ideal in $C(X)$.

Proof. Since $A$ is disjoint from $Z_{f} \cap S_{f}$, which is a zero set of $S_{f}$, there exists a function $h \in C\left(S_{f}\right)$ such that $h[A]=0$ while $h$ is identically 1 on a neighborhood of $Z_{f} \cap S_{f}$. We may extend $h$ continuously to all $X$ by assigning it the value 1 outside of $S_{f}$. Since $A \subset Z_{h}, Z_{h}$ is not compact and therefore $h$ belongs to some free maximal ideal $M^{p}$; however, the fact that $Z_{h} \cap Z_{f}=\emptyset$ implies $f \notin M^{p}$.

THEOREM. The following statements are equivalent.

(1) $C_{K}(X)$ is the intersection of the free maximal ideals in $C(X)$.

(2) If $f$ belongs to the intersection of the free maximal ideals in $C(X)$, then $S_{f}$ is complete in some uniform structure. 
(3) If $O$ is any open, locally compact, $\sigma$-compact subset of $X$ and $c l_{X} O$ is not compact, then for some $q \in \beta X-X, M^{q}$ is hyper-real and $q \in c l_{\beta X} O$.

(4) If $O$ is any open, locally compact, $\sigma$-compact subset of $X$, then $c l_{X} O$ is either compact or admits an unbounded continuous real-valued function.

Proof. (1) implies (2). Obvious.

(2) implies (3). Let $O=\bigcup\left\{K_{n}: n \in N\right\}$, where each $K_{n}$ is compact and where $O$ is open in $X$ and locally compact. Then,

$$
\beta X-O\left(=\cap\left\{\beta X-K_{n}: n \in N\right\}\right)
$$

is a closed $G_{\delta}$; hence it equals $Z_{\hat{g}}$ for some $\hat{g} \in C(\beta X)$. If we let $g$ denote the restriction to $X$ of $\hat{g}$, we observe that $O=X-Z_{g}$. If $g$ belongs to the intersection of the free maximal ideals, (2) implies that $c l_{X} O$ is complete. Since a complete space is either compact or admits an unbounded continuous function, our lemma implies that $c l_{X} O$ is compact. This contradicts the hypothesis of (3); hence we conclude that $g$ fails to belong to some free maximal ideal.

Next, we observe that if $p \in \beta X-X$ and $M^{p}$ is real, $p \in c l_{\beta X} Z_{g}$. For in this case we have that for each $n \in N$, the zero set $\{x:|g(x)| \leqq 1 / n\}$ belongs to the associated $Z$-ultrafilter $Z\left[M^{p}\right]$, and therefore that $Z_{g}$, being the intersection of this countable family, also belongs, i.e., $p \in c l_{\beta X} Z_{g}$. Thus, $g$ must belong to each free maximal ideal that is real. Hence, if $c l_{X} O\left(=S_{g}\right)$ is not compact, $g$ must fail to belong to some hyper-real maximal ideal, $M^{q}$. Thus, since $q \notin c l_{\beta X} Z_{g}$, it follows that $q \in c l_{\beta X} O$.

(3) implies (4). If $c l_{X} O$ is not compact, and $q \in c l_{\beta X} O$, where $M^{q}$ is hyper-real, we infer that for some $h \in C(X)$ and for all $n \in N$ the sets $c l_{\beta X}\{x: h(x) \geqq n\}$ are neighborhoods of $q$. Since each of these sets meets $O$, the restriction to $c l_{X} O$ of $h$ is the desired unbounded function.

(4) implies (1). If $f$ belongs to the intersection of the free maximal ideals, then $X-Z_{f}$, satisfies the hypotheses of (4). Thus, if $f \notin C_{K}(X)$, then $S_{f}$ must admit an unbounded continuous function. But the existence of such a function implies the existence in $X-Z_{f}$ of a closed, non-compact and $C$-embedded subset of $S_{f}$. An application of our lemma yields the desired contradiction and completes the proof.

REMARk. The referee has pointed out that the equivalence of (1), (2), and (4) can be proved without constructing $\beta X$.

The result of Kohls as well as that obtained in [2] follows directly from (2), as does the following corollary.

COROLLARY. If $X$ is peripherally Lindelof (every co-zero set has a boundary which is Lindelof) then the intersection of the free maximal ideals in $C(X)$ is $C_{K}(X)$. 


\section{References}

[1] L. Gillman and M. Jerison, Rings of Continuous Functions, (Van Nostrand Co., Princeton, New Jersey, 1960).

[2] S. M. Robinson, 'The intersection of the free maximal ideals in a complete space', Proceedings of the American Mathematical Society, 17, April 1966, pp. 468-469.

The Cleveland State University 